University of Wollongong

Research Online

Australian Institute for Innovative Materials -

Papers

Australian Institute for Innovative Materials

$1-1-2012$

Temperature and frequency dependent giant magnetodielectric coupling in DyMn0.33Fe0.6703

\author{
Fang Hong \\ University of Wollongong, fh640@uowmail.edu.au \\ Zhenxiang Cheng \\ University of Wollongong, cheng@uow.edu.au \\ Xiaolin Wang \\ University of Wollongong, xiaolin@uow.edu.au
}

Follow this and additional works at: https://ro.uow.edu.au/aiimpapers

Part of the Engineering Commons, and the Physical Sciences and Mathematics Commons

Research Online is the open access institutional repository for the University of Wollongong. For further information contact the UOW Library: research-pubs@uow.edu.au 


\title{
Temperature and frequency dependent giant magnetodielectric coupling in DyMn0.33Fe0.6703
}

\author{
Abstract \\ Perovskite DyMn0.33Fe0.6703 experiences a paramagnetism-antiferromagnetism transition at $450 \mathrm{~K}$ and \\ spin reorientation at $290 \mathrm{~K}$. Magnetodielectric properties were studied around the spin reorientation \\ transition. Both giant positive and giant negative magnetodielectric coupling (MDC) were observed near \\ room temperature. The MDC shows strong temperature and frequency dependence, and the sign changes \\ from positive to negative when magnetic state transits from a canted antiferromagnetic state to a \\ collinear antiferromagnetic state. Possible mechanisms are proposed based on the Maxwell-Wagner \\ model, phase transition, the magnetoresistance effect, and spin-phonon coupling.

\section{Keywords} \\ dependent, frequency, temperature, coupling, magnetodielectric, giant, dymn0, 67o3, 33fe0 \\ Disciplines \\ Engineering | Physical Sciences and Mathematics

\section{Publication Details} \\ Hong, F., Cheng, Z. \& Wang, X. (2012). Temperature and frequency dependent giant magnetodielectric \\ coupling in DyMn0.33Fe0.6703. Journal of Applied Physics, 112 (1), 013920-1-013920-5.
}




\section{AIP Apoinied Phisis}

\section{Temperature and frequency dependent giant magnetodielectric coupling in DyMn0.33Fe0.6703}

Fang Hong, Zhenxiang Cheng, and Xiaolin Wang

Citation: J. Appl. Phys. 112, 013920 (2012); doi: 10.1063/1.4736543

View online: http://dx.doi.org/10.1063/1.4736543

View Table of Contents: http://jap.aip.org/resource/1/JAPIAU/v112/i1

Published by the American Institute of Physics.

\section{Related Articles}

Enhanced magneto-impedance in Fe73.5Cu1Nb3Si13.5B9 ribbons from laminating with magnetostrictive terfenol-D alloy plate

Appl. Phys. Lett. 101, 251914 (2012)

Extensive study of giant magnetoresistance properties in half-metallic Co2(Fe,Mn)Si-based devices Appl. Phys. Lett. 101, 252408 (2012)

Anisotropic magnetoresistance in topological insulator Bi1.5Sb0.5Te1.8Se1.2/CoFe heterostructures AIP Advances 2, 042171 (2012)

Angular-dependences of giant in-plane and interlayer magnetoresistances in Bi2Te3 bulk single crystals Appl. Phys. Lett. 101, 152107 (2012)

Magnetic field-dependent effective microwave properties of microwire-epoxy composites

Appl. Phys. Lett. 101, 152905 (2012)

\section{Additional information on J. Appl. Phys.}

Journal Homepage: http://jap.aip.org/

Journal Information: http://jap.aip.org/about/about_the_journal

Top downloads: http://jap.aip.org/features/most_downloaded

Information for Authors: http://jap.aip.org/authors

\section{ADVERTISEMENT}

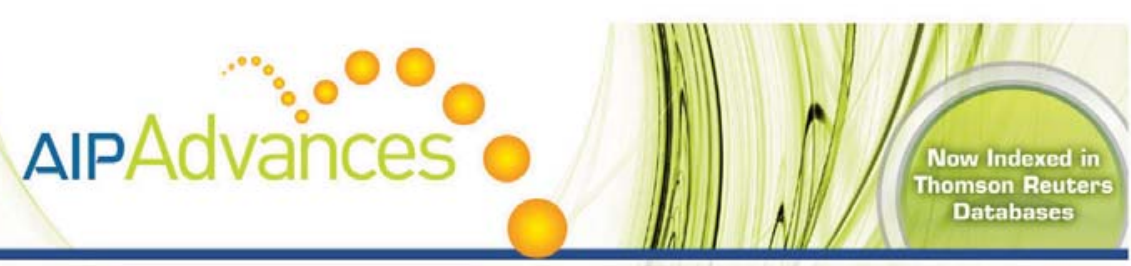

\section{Explore AIP's open access journal: •Rapid publication \\ - Article-level metrics \\ Post-publication rating and commenting}




\title{
Temperature and frequency dependent giant magnetodielectric coupling in $\mathrm{DyMn}_{0.33} \mathrm{Fe}_{0.67} \mathrm{O}_{3}$
}

\author{
Fang Hong, Zhenxiang Cheng, ${ }^{\text {a) }}$ and Xiaolin Wang \\ Institute for Superconducting and Electronic Materials, University of Wollongong, Australia
}

(Received 14 February 2012; accepted 9 June 2012; published online 12 July 2012)

\begin{abstract}
Perovskite $\mathrm{DyMn}_{0.33} \mathrm{Fe}_{0.67} \mathrm{O}_{3}$ experiences a paramagnetism-antiferromagnetism transition at $450 \mathrm{~K}$ and spin reorientation at $290 \mathrm{~K}$. Magnetodielectric properties were studied around the spin reorientation transition. Both giant positive and giant negative magnetodielectric coupling (MDC) were observed near room temperature. The MDC shows strong temperature and frequency dependence, and the sign changes from positive to negative when magnetic state transits from a canted antiferromagnetic state to a collinear antiferromagnetic state. Possible mechanisms are proposed based on the Maxwell-Wagner model, phase transition, the magnetoresistance effect, and spin-phonon coupling. (C) 2012 American Institute of Physics. [http://dx.doi.org/10.1063/1.4736543]
\end{abstract}

\section{INTRODUCTION}

Dielectric materials are common materials which have wide applications in energy storage, electronic switches, sensors, and actuators. To develop next generation multifunctional devices, effective control of more degrees of freedom beyond electric charge should be given a priority. Considerable work has been done to control the dielectric property by magnetic methods, ${ }^{1-4}$ the so-called magnetodielectric coupling (MDC) effect. Most MDC effects appear around the magnetic phase transition temperature, at which dielectric abnormality can be observed and modified by external magnetic fields. These effects are notably found in some manganites. In $\mathrm{BiMnO}_{3}$, the dielectric constant is suppressed around $100 \mathrm{~K}$, where ferromagnetic (FM) ordering occurs ${ }^{5}$ and a giant negative MDC effect could be induced. For spin frustrated perovskite $\mathrm{TbMnO}_{3}$ (Pbnm space group), ${ }^{6}$ an obvious peak in the temperature dependence of the $c$-axis dielectric constant is found at $27 \mathrm{~K}$, where the sinusoidal antiferromagnetic (AFM) magnetic modulation wave vector is locked at a constant value. Magnetic field can shift the dielectric peak slightly around $27 \mathrm{~K}$ and induce an extra dielectric peak at lower temperature. In metastable orthorhombic $\mathrm{HoMnO}_{3}$ and $\mathrm{YMnO}_{3}{ }^{7}$ the $\mathrm{MDC}$ effect occurs below their incommensurate AFM transition temperature of $42 \mathrm{~K}$. Apart from these manganites, the MDC effect can be found in other systems, such as $\mathrm{SeCuO}_{3},{ }^{8} \mathrm{TeCuO}_{3},{ }^{8} \mathrm{MCr}_{2} \mathrm{O}_{4}$ (Ref. 9) $(\mathrm{M}=\mathrm{Mn}, \mathrm{Co}$, and $\mathrm{Ni})$, and $\mathrm{Bi}_{2} \mathrm{Mn}_{4 / 3} \mathrm{Ni}_{2 / 3} \mathrm{O}_{6}$, although their MDC values are very small. Colossal magnetoresistance (MR) materials, such as $\mathrm{EuNbO}_{2} \mathrm{~N}$ (Ref. 11) and $\mathrm{HgCr}_{2} \mathrm{~S}_{4}$, ${ }^{12}$ also show significant magnetodielectric response at low temperature. To achieve practical applications, it would be ideal to find materials with MDC effects near room temperature. In addition, the MDC effect should be strong. Here, we present both giant positive and giant negative MDC in perovskite $\mathrm{DyMn}_{0.33} \mathrm{Fe}_{0.67} \mathrm{O}_{3}$ near room temperature, $290 \mathrm{~K}$, at which spin reorientation occurs.

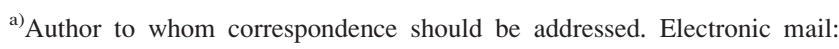
cheng@uow.edu.au.
}

\section{EXPERIMENT}

Polycrystalline sample of $\mathrm{DyMn}_{0.33} \mathrm{Fe}_{0.67} \mathrm{O}_{3}$ was made by the traditional solid state reaction method with $\mathrm{Dy}_{2} \mathrm{O}_{3}$ (99.9\%), $\mathrm{MnCO}_{3}(99.9 \%)$, and $\mathrm{Fe}_{2} \mathrm{O}_{3}(99.9 \%)$ powder bought from Sigma-Aldrich. Stoichiometric amounts of raw oxide powder were weighed carefully and mixed in an agate mortar, followed by pressing into pellets $15 \mathrm{~mm}$ in diameter at $20 \mathrm{MPa}$. Samples were calcined at $950^{\circ} \mathrm{C}$ for $10 \mathrm{~h}$ and sintered at $1440^{\circ} \mathrm{C}$ for $6 \mathrm{~h}$. The crystal structure of the sample was examined by $\mathrm{x}$-ray diffraction (XRD, Model: GBC MMA), using $\mathrm{Cu} K \alpha$ radiation at $\lambda=1.54056 \AA$. The Rietveld refinement calculations were conducted via FULLPROF software. The magnetic measurements were carried out using a $14 \mathrm{~T}$ physical properties measurement system (PPMS), equipped with a vibrating sample magnetometer (VSM), in magnetic fields up to $5 \mathrm{~T}$, over a wide temperature range from 5 to $340 \mathrm{~K}$ in standard mode, and from $310 \mathrm{~K}$ to $750 \mathrm{~K}$ in oven mode. Pt electrodes were deposited on both sides of the thin pellet by magnetic scattering coating for dielectric meausrement. An Agilent 4294 A impedance analyser was employed for dielectric property measurements, scanning from $1 \mathrm{kHz}$ to $1 \mathrm{MHz}$, while the temperature and applied magnetic fields were controlled by PPMS. A room temperature resistance measurement was carried out with a multimeter, and it showed resistivity of $\sim 10^{7} \Omega \mathrm{cm}$.

\section{RESULTS AND DISCUSSION}

Figure 1 shows the $\mathrm{x}$-ray powder diffraction pattern and Rietveld refinement results at room temperature. All diffraction peaks can be assigned to the single phase orthorhombic structure with space group Pnma, and no detectable impurity phase is present. The quality of refinement is determined by refinement parameter $\mathrm{R} p=10.2 \%$ and variance $\chi^{2}=1.9$. The calculated lattice parameters are $a=5.6237(10) \AA$, $b=7.5602(13) \AA$, and $c=5.2912(9) \AA$.

The temperature dependence of the magnetic moment of $\mathrm{DyMn}_{0.33} \mathrm{Fe}_{0.67} \mathrm{O}_{3}$ is shown in Figure 2(a). There are two transitions that can be clearly indentified. One is the transition from paramagnetism (PM) to canted AFM, with a weak 


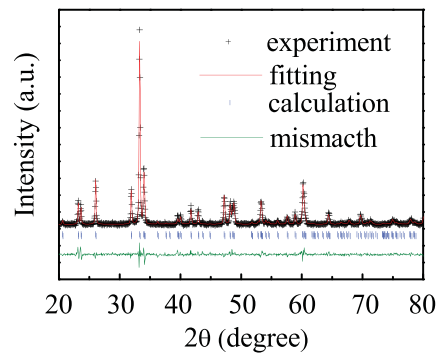

FIG. 1. XRD refinement result of $\mathrm{DyMn}_{0.33} \mathrm{Fe}_{0.67} \mathrm{O}_{3}$ with $\mathrm{R} p=9.9 \%$ (star symbols, measured, and solid line, fitted). The difference between the measured and Rietveld refined spectra is plotted with a slight downshift for clarity. The short vertical solid lines are guides for the eyes to mark the corresponding Bragg positions.

ferromagnetic (WFM) component around $450 \mathrm{~K}$, while the other is the transition from a canted AFM (WFM) to a collinear AFM around $290 \mathrm{~K}$ as the temperature decreases. CurieWeiss fitting was conducted above $450 \mathrm{~K}$, as displayed in Figure 2(b), where the sample shows intrinsic paramagnetic features, giving a Curie-Weiss temperature of $-79 \mathrm{~K}$, Curie constant of 15.6 , and effective moment of $11.2 \mu_{\mathrm{B}}$. The negative Curie-Weiss temperature suggests the antiferromagnetic transition around $450 \mathrm{~K}$. The theoretical value of maximum moment for $\mathrm{DyMn}_{0.33} \mathrm{Fe}_{0.67} \mathrm{O}_{3}$ is $11.4 \mu_{\mathrm{B}}$ if all ions are in the high spin state $\left(4.9 \mu_{\mathrm{B}} / \mathrm{Mn}^{3+}, 5.9 \mu_{\mathrm{B}} / \mathrm{Fe}^{3+}\right.$, and $\left.9.9 \mu_{\mathrm{B}} / \mathrm{Dy}^{3+}\right)$. The close values of the theoretical and the experimental moments indicate that all magnetic ions are in the high spin state. The increasing moment below this transition indicates that the antiferromagnetism is not pure but has a weak ferromagnetic component. Generally, in G-type antiferromagnetic order, a WFM component could be induced along the $c$ axis because of the Dzyaloshinsky-Moriya interaction, if spins are normal to $b$ axis in the $a b$ plane. ${ }^{13}$ The possibility of FM clusters can be easily excluded because the magnetic hysteresis loop measured at $5 \mathrm{~K}$ shows typical paramagnetic/ antiferromagnetic behaviour. A similar antiferromagnetic transition can also be observed in $\mathrm{DyFeO}_{3} .{ }^{14}$ The sudden decrease in the magnetic moment at $290 \mathrm{~K}$ is likely to be the result of the disappearance of the WFM component. Below it, the sample is in the pure AFM state, as confirmed by the magnetic hysteresis loop at $5 \mathrm{~K}$. Hence, this transition is a spin reorientation caused by the change in the highly temperature sensitive magnetic anisotropy, which is similar to the case of $\mathrm{DyFeO}_{3} \cdot{ }^{14}$ The continuous increase in the magnetic moment at low temperature is due to paramagnetic behaviour of $\mathrm{Dy}^{3+}$. The magnetic property is consistent with the previous work. $^{15}$
To investigate coupling between the magnetic field and the dielectric property, dielectric constants and loss were collected in different magnetic fields at $310 \mathrm{~K}, 285 \mathrm{~K}$, and $250 \mathrm{~K}$, respectively, as shown in Figures 3(a), 3(b), and 3(c). Obvious up/down shifts in the dielectric constant can be observed in the frequency dependence of the dielectric constants when measurements were carried out in various magnetic fields, indicating the occurrence of magnetodielectric coupling. The strength of magnetodielectric coupling is determined by the MDC constant in the form of $\left(\varepsilon_{\mathrm{H}}-\varepsilon_{\mathrm{H}=0}\right) / \varepsilon_{\mathrm{H}=0}$, which is calculated and given in Figures 4(a)-4(c). It is clear that the MDC is extremely significant, especially in high magnetic field. At $310 \mathrm{~K}$, we found a positive MDC constant as large as 53\% (0.53) around $2 \times 10^{4} \mathrm{~Hz}$. At the low frequency range, the MDC constant approaches zero when the frequency decreases, indicating that mobile charge does not contribute to the MDC effect too much. Just below the spin reorientation temperature, at $285 \mathrm{~K}$, an opposite MDC effect occurs, giving a large value of $-35 \%$ around $1 \times 10^{4} \mathrm{~Hz}$. The MDC constant also approaches zero when the frequency is close to $0 \mathrm{~Hz}$, confirming the negligible effect of mobile charge on MDC. Maximum MDC can be observed at the conductivity cut-off frequency 1/RC (where $\mathrm{R}$ and $\mathrm{C}$ are the resistance and capacitance of the equal circle, respectively). ${ }^{16}$ When the temperature cools down from the spin reorientation temperature, this negative MDC effect becomes much stronger, reaching $-70 \%$ around $5 \times 10^{3} \mathrm{~Hz}$ at $250 \mathrm{~K}$, as shown in Figure 4(c). Hence, the MDC effect is strongly temperature dependent. On the other hand, clear frequency dependence of MDC can be well observed.

Up to now, there are three proposed mechanisms to explain the MDC effect. One is based on intrinsic multiferroic relaxor behaviour, ${ }^{17}$ one is based on the Maxwell-Wagner model, ${ }^{16}$ and one is based on spin-phonon interaction. Although $\mathrm{DyMnO}_{3}$ and $\mathrm{DyFeO}_{3}$ also show multiferroic features, their multiferroic properties can only appear at very low temperature, below $18 \mathrm{~K}$ (Ref. 18) and $3.5 \mathrm{~K},{ }^{19}$ because of the inverse Dzyaloshinsky-Moriya interaction and exchange striction, respectively. Hence, near room temperature, the multiferroic relaxor based mechanism is not appropriate for our result. However, the giant MDC can be tentatively explained by the Maxwell-Wagner model at low frequency range below the cutoff frequency $1 / \mathrm{RC}$. To better understand these unique phenomena, we present the frequency dependence of the dynamic magnetoloss $(\mathrm{ML}), \mathrm{ML}=\left(\operatorname{Loss}_{\mathrm{H}}-\operatorname{Loss}_{\mathrm{H}=0}\right) / \operatorname{Loss}_{\mathrm{H}=0}$, as shown in Figures 4(a)-4(c). The significant change of ML can be observed as increasing frequency, and the ML shows the opposite tendency to that of MDC. Considering the ML effect and
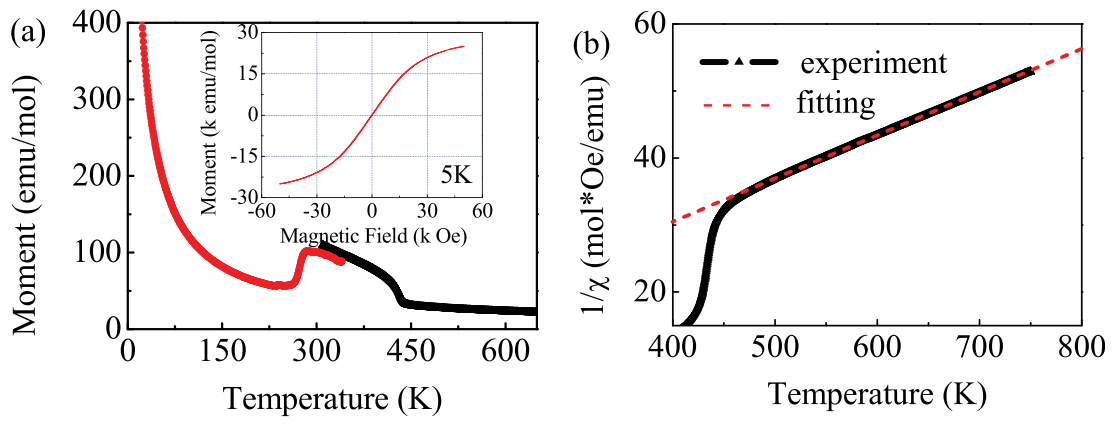

FIG. 2. (a) Temperature dependence of magnetic moment from $10 \mathrm{~K}$ to $600 \mathrm{~K}$ (black closed triangles: standard mode; red closed circles: oven mode). Inset: magnetic hysteresis loop measured at $5 \mathrm{~K}$. (b) Inverse DC susceptibility versus temperature as measured in an external field of 1000 Oe for the paramagnetic state of $\mathrm{DyMn}_{0.33} \mathrm{Fe}_{0.67} \mathrm{O}_{3}$ above $450 \mathrm{~K}$ (dashed line: Curie-Weiss fit; triangles: the inverse curve from experimental data). 
(a)

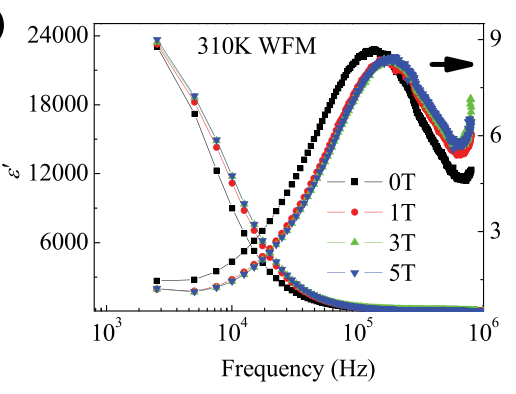

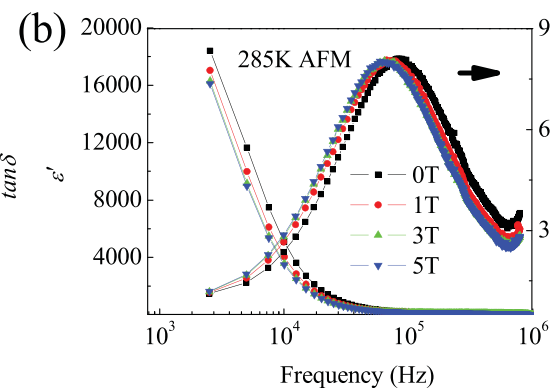

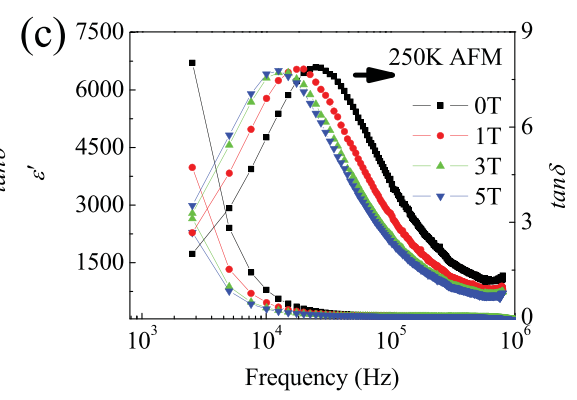

FIG. 3. Frequency dependence of dielectric constant, loss at $310 \mathrm{~K}, 285 \mathrm{~K}$, and $250 \mathrm{~K}$, respectively.
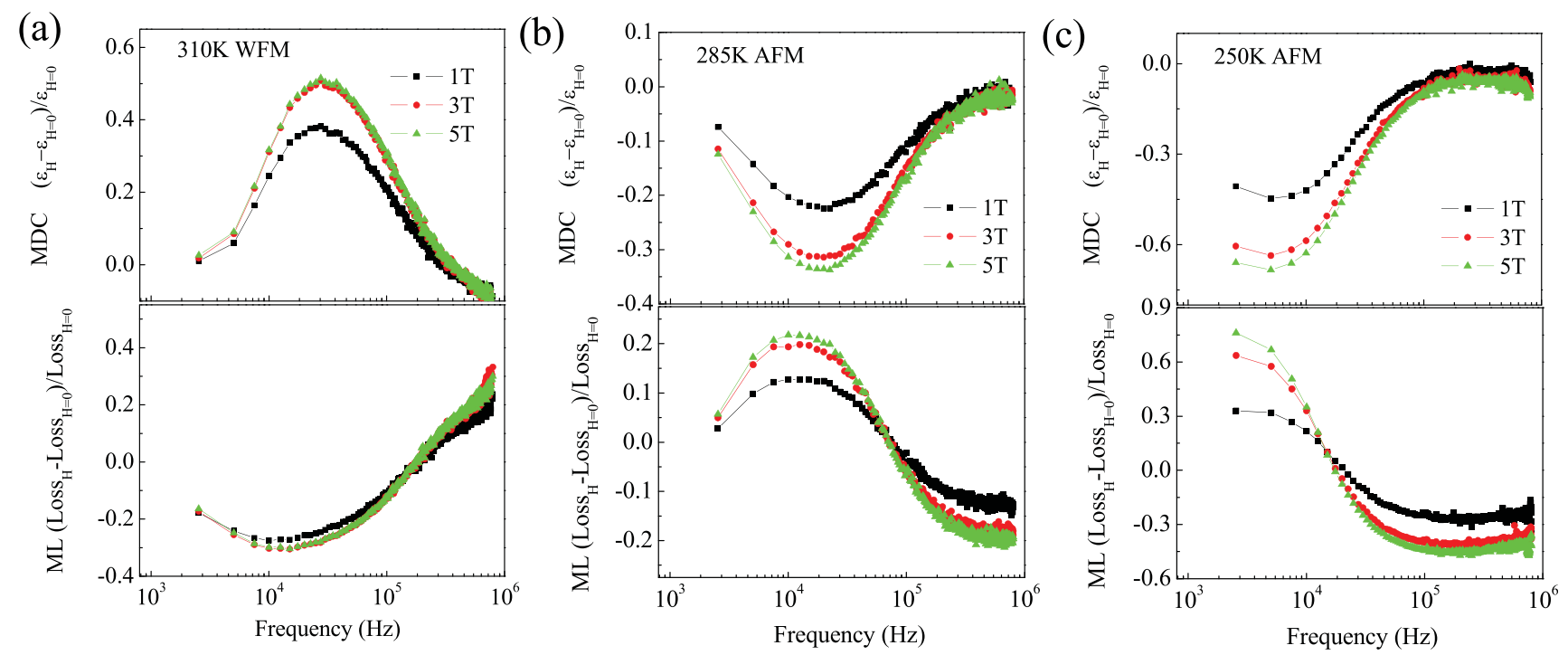

FIG. 4. Frequency dependence of MDC constant and dynamic ML at $310 \mathrm{~K}, 285 \mathrm{~K}$, and $250 \mathrm{~K}$, respectively.

the result in Ref. 16, we could assign this MDC effect to the MR related MDC effects. Above the spin reorientation temperature of $290 \mathrm{~K}$, such as at $310 \mathrm{~K}$, the material shows WFM, but the applied magnetic field suppresses the spin fluctuation in the WFM state. ${ }^{20,21}$ This results in a negative MR and favours a positive MDC effect according to the Maxwell-Wagner model, similar to the positive MDC effect that occurs in $\mathrm{EuNbO}_{2} \mathrm{~N}$ below the ferromagnetic transition temperature. ${ }^{11}$ During our experiment measurement, dynamic frequency dependent resistances were obtained. Figure 5 presents the dynamic magnetoresistance in form of $M R=\left(R_{H}-R_{H=0}\right) / R_{H=0}$. A negative $M R$ effect is observed at $310 \mathrm{~K}$ as shown in Figure 5(a). The result confirms our assumption. On the contrary, when the temperature is $285 \mathrm{~K}$ or $250 \mathrm{~K}$, the material experiences a magnetic phase transition from WFM to AFM. The MDC may be contributed by the following possible factors: first, MR effect induced by spin polarized tunnelling between charge depleted grain boundaries; ${ }^{20}$ second, the intrinsic magnetoelectric effect due to magnetostriction; ${ }^{22}$ third, the spin pair correlation, as explained in the phenomenal model. ${ }^{23}$ In our case, the magnetostriction effect should be very small and negligible, as the sample is not a typical magnetostriction material. In addition, the spin pair correlation cannot induce such a giant MDC effect. Hence, the most likely factor is the contribution by MR effect induced by spin polarized (a)

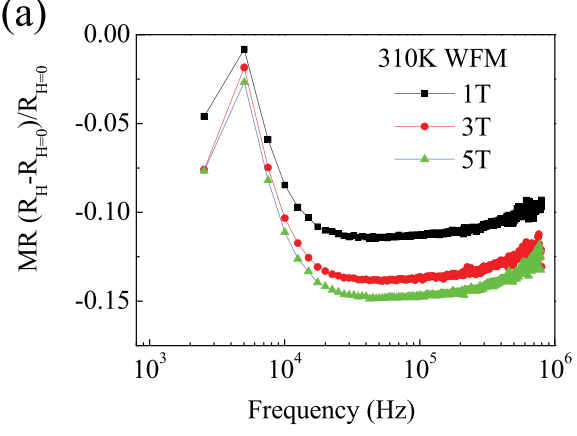

(b)

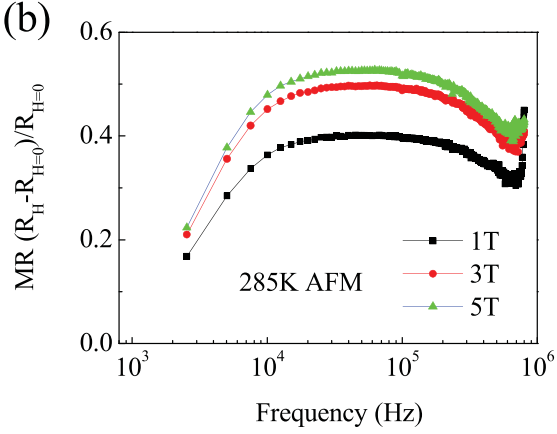

(c)

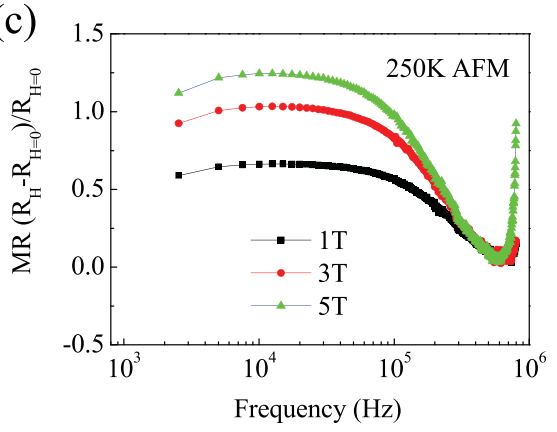

FIG. 5. Frequency dependence of dynamic MR at $310 \mathrm{~K}, 285 \mathrm{~K}$, and $250 \mathrm{~K}$, respectively. 
tunnelling, despite the AFM property. This is proved by the positive MR effects are observed at $280 \mathrm{~K}$ and $250 \mathrm{~K}$ as shown in Figures 5(b) and 5(c), which could induce a negative MDC according to the Maxwell-Wagner model. In the light of this, the MDC at low frequency range below the cut-off frequency $1 / \mathrm{RC}$ is related to space charge effect, which can be further confirmed by the strong frequency dependence and MR effect. For the case at high frequency range above the cut-off frequency $1 / \mathrm{RC}$, the MDC effect becomes weaker, which should due to the absence of space charge effect. Different magnetic states will affect the strength and mode of spin-phonon interaction and dielectric constant is consequently modified. ${ }^{8,24}$ Similar effect is observed in $\mathrm{Mn}_{3} \mathrm{O}_{4}$, in which opposite MDC effects happen above and below magnetic transition temperature. ${ }^{25}$ Hence, spin-phonon interaction is supposed to be responsible for the MDC at high frequency range.

\section{CONCLUSIONS}

In summary, we have studied the phase transitions and MDC effect in $\mathrm{DyMn}_{0.33} \mathrm{Fe}_{0.67} \mathrm{O}_{3}$. Two magnetic phase transitions are found at $290 \mathrm{~K}$ and $450 \mathrm{~K}$, corresponding to spin reorientation and the antiferromagnetism-paramagnetism transition, respectively. MDC show strong temperature and frequency dependence. Giant positive and negative MDC effects are observed in the vicinity of the spin reorientation temperature. Magnetoresistance based Maxwell-Wagner model is employed to explain the MDC at low frequency range below the cut-off frequency $1 / \mathrm{RC}$ and spin-phonon interaction is responsible for the MDC at high frequency range above the cutoff frequency $1 / \mathrm{RC}$. These effects make it promising to produce multifunctional devices based on such kinds of materials.

\section{ACKNOWLEDGMENTS}

Zhenxiang Cheng thanks the Australian Research Council for support through a Future Fellowship (FT 0990287). The authors also thank Dr. Tania Silver for her kind help in revision of the manuscript.
${ }^{1}$ T. Bonaedy, Y. S. Koo, K. D. Sung, and J. H. Jung, Appl. Phys. Lett. 91, 132901 (2007).

${ }^{2}$ M. A. Subramanian, T. He, J. Z. Chen, N. S. Rogado, T. G. Calvarese, and A. W. Sleight, Adv. Mater. 18, 1737 (2006).

${ }^{3}$ P. Liu, Z. X. Cheng, Y. Du, and X. L. Wang, J. Phys. D: Appl. Phys. 43, 325002 (2010).

${ }^{4}$ Z. X. Cheng, H. Shen, J. Y. Xu, P. Liu, S. J. Zhang, J. L. Wang, X. L. Wang, and S. X. Dou, J. Appl. Phys. 111, 034103 (2012).

${ }^{5}$ T. Kimura, S. Ishihara, H. Shintani, T. Arima, K. T. Takahashi, K. Ishizaka, and Y. Tokura, Phys. Rev. B 68, 060403 (2003).

${ }^{6}$ T. Kimura, T. Goto, H. Shintani, K. Ishizaka, T. Arima, and Y. Tokura, Nature 426, 55 (2003).

${ }^{7}$ B. Lorenz, Y. Q. Wang, Y. Y. Sun, and C. W. Chu, Phys. Rev. B 70, 212412 (2004).

${ }^{8}$ G. Lawes, A. P. Ramirez, C. M. Varma, and M. A. Subramanian, Phys. Rev. Lett. 91, 257208 (2003).

${ }^{9}$ N. Mufti, A. A. Nugroho, G. R. Blake, and T. T. M. Palstra, J. Phys.: Condens. Matter 22, 075902 (2010).

${ }^{10}$ J. B. Claridge, H. Hughes, C. A. Bridges, M. Allix, M. R. Suchomel, H. Niu, X. Kuang, M. J. Rosseinsky, N. Bellido, D. Grebille, O. Perez, C. Simon, D. Pelloquin, S. J. Blundell, T. Lancaster, P. J. Baker, F. L. Pratt, and P. S. Halasyamani, J. Am. Chem. Soc. 131, 14000 (2009).

${ }^{11}$ A. B. N. Jorge, J. Oró-Solé, A. M. Bea, N. Mufti, T. T. M. Palstra, J. A. Rodgers, J. P. Attfield, and A. Fuertes, J. Am. Chem. Soc. 130, 12572 (2008).

${ }^{12}$ S. Weber, P. Lunkenheimer, R. Fichtl, J. Hemberger, V. Tsurkan, and A. Loidl, Phys. Rev. Lett. 96, 157202 (2006).

${ }^{13}$ Y. H. Huang, M. Karppinen, N. Imamura, H. Yamauchi, and J. B. Goodenough, Phys. Rev. B 76, 174405 (2007).

${ }^{14}$ A. Berton and B. Sharon, J. Appl. Phys. 39, 1367 (1968).

${ }^{15}$ F. Hong, Z. Cheng, H. Zhao, H. Kimura, and X. Wang, Appl. Phys. Lett. 99, 092502 (2011).

${ }^{16}$ G. Catalan, Appl. Phys. Lett. 88, 102902 (2006).

${ }^{17}$ J. Hemberger, P. Lunkenheimer, R. Fichtl, H.-A. K. von Nidda, V. Tsurkan, and A. Loidl, Nature 448, E5 (2007).

${ }^{18}$ Y. Tokura and S. Seki, Adv. Mater. 22, 1554 (2010).

${ }^{19}$ Y. Tokunaga, S. Iguchi, T. Arima, and Y. Tokura, Phys. Rev. Lett. 101, 097205 (2008).

${ }^{20}$ H. Y. Hwang, S. W. Cheong, N. P. Ong, and B. Batlogg, Phys. Rev. Lett. 77, 2041 (1996).

${ }^{21}$ M. F. Hundley, J. J. Neumeier, R. H. Heffner, Q. X. Jia, X. D. Wu, and J. D. Thompson, J. Appl. Phys. 79, 4535 (1996).

${ }^{22}$ G. Srinivasan, E. T. Rasmussen, B. J. Levin, and R. Hayes, Phys. Rev. B 65, 134402 (2002).

${ }^{23}$ T. Katsufuji and H. Takagi, Phys. Rev. B 69, 064422 (2004).

${ }^{24}$ G. A. Samara and P. M. Richards, Phys. Rev. B 14, 5073 (1976).

${ }^{25}$ R. Tackett, G. Lawes, B. C. Melot, M. Grossman, E. S. Toberer, and R. Seshadri, Phys. Rev. B 76, 024409 (2007). 